\title{
Self-Stratifying Particulate Coating for Robust Superhydrophobic and Latex-Repellent Surface
}

\author{
Sulaiman Hajeesaeh ${ }^{1,2,3}$, Sobiroh Kariyo², Nantakan Muensit ${ }^{1,3}$, Chalongrat Daengngam ${ }^{1,3, *}$ \\ ${ }^{1}$ Department of Physics, Faculty of Science, Prince of Songkla University, Songkhla 90112, Thailand \\ ${ }^{2}$ Department of Research and Development, Faculty of Science and Technology, Fatoni University, Pattani 94160, Thailand \\ ${ }^{3}$ Center of Excellence in Nanotechnology for Energy, Prince of Songkla University, Songkhla 90112, Thailand
}

Received: $19 / 05 / 2020$

Accepted: 08/07/2020

Published: 20/09/2020

\begin{abstract}
A technique for preparing superhydrophobic and natural latex-repellent surface requires at least two fabrication components: surface roughness, and surface layer with low free energy. Here, multiscale surface roughness in micro-/nanoscales with low surface energy can be simultaneously achieved through the deposition of fluoroalkyl-functionalized silica aggregates. However, the mechanical durability of such film remains problematic. Therefore, third component such as polymer binder was incorporated carefully to improve adhesion between filmsubstrate interface without deteriorating surface roughness and surface energy. In this work, we employed self-stratifying coating technique to induce vertical phase separation between particles and polymer during film drying, such that the silica aggregates densely accumulated on the top surface, while polymer binder concentrated near the film bottom. The governing transports during film stratification process involve diffusion and convection driven by evaporation. Thus, this research focused on the effect of drying temperature and evaporation rate on the anti-wetting performance of the coating. The results showed that the liquid-repellent properties of the surface improve with increasing drying temperature, indicating the convection-dominated transport that induced substantial particle trap at the film surface. With polymer binder added, the coatings still showed decent superhydrophobic and natural latex-repellent properties with maximum contact angles $166.4^{\circ} \pm 0.6^{\circ}$ and $157.5^{\circ} \pm 0.5^{\circ}$, as well as minimum sliding angles $2.7^{\circ} \pm 0.3^{\circ}$ and $2.9^{\circ} \pm 0.2^{\circ}$ for water and natural latex respectively. Also, AFM result revealed that significant surface roughness of $581 \pm 18 \mathrm{~nm}$ was still achievable even at high blending mass ratio of polymer binder up to half of the silica weight.
\end{abstract}

Keywords: Superhydrophobic, Natural latex-repellent surface, Multiscale roughness, Self-stratifying coating

\section{Introduction}

It is well known that the extreme liquid-repellent properties of surface are the synergistic effect of surface morphology and chemical compositions (1). Surface free energy of coating films can be lowered by mean of surface functionalization with hydrophobic molecules, and the hydrophobicity can be further enhanced by surface asperities to reach superhydrophobic state. Therefore, both surface topographical roughness and the outmost functional groups play a crucial role in producing such superior liquid-repellent properties. Furthermore, surface roughness in multiple length scale has been proven as the key to achieve more stable Cassie-Baxter non-wetting state (2). In our previous research, we also demonstrated that tipple-scale surface roughness, obtained from silica aggregates functionalized with fluoroalkylsilane molecules, were superior to from stable extreme anti-wetting surface that can repel water or even highly adhesive liquid like concentrated natural latex (3). As the film was mainly composed of functionalized nanoparticles, however, its poor mechanical durability remains a major drawback for real uses. Also, it is quite a contradiction to expect non-sticky functionalized particles to adhere tightly on a substrate. The strategy to improve the adhesion between superhydrophobic film and substrate requires addition of fluoro-containing polymer binder, which allows good dispersion of fluoroalkyl functionalized silica nanoparticles into the polymer matrix. Nonetheless, it remains quite complicated to maintain the surface roughness and the topmost functional groups of final film, as polymer binder tends to swamp the surface.

Therefore, an asymmetric particle distribution induced by selfstratification during film drying is introduced, in order to produce spatially controlled polymer blending, which does not destroy superhydrophobic features (4). As particulate coating dries, diffusion and convection transports determine the final particle distribution inside the film. Particles tend to accumulate more on coating surface when the convection dominates, i.e. high evaporation rate, so higher surface roughness can be expected. On the other hand, if the diffusion dominates, particles and polymer

Corresponding author: Chalongrat Daengngam, (a) Department of Physics, Faculty of Science, Prince of Songkla University, Songkhla 90112, Thailand. (b) Center of Excellence in Nanotechnology for Energy, Prince of Songkla University, Songkhla 90112, Thailand. E-mail: chalongrat.d@psu.ac.th. 
will blend uniformly causing smoother surface, unfavorable for superhydrophobic properties.

To accomplish the aforesaid idea of stratified drying, a 1D vertical model of particle transport in film during drying formation, developed by Routh and Russel, was considered (5). It can be used to predict skin formation occurring for non-uniform vertical drying combined with wet sintering $(6,7)$. This model approach can be employed to determine whether the particle transport is controlled by diffusion or convection through the dimensionless Peclet number, which is defined by the following expression

$$
P_{e}=\frac{E H_{0}}{D_{0}}
$$

where $P_{e}$ is the Peclet number. $E$ is the film evaporation rate measured from the speed of the descending surface. $H_{0}$ is the film initial thickness, and $D_{0}$ is the diffusion coefficient of particle, which is related to the particle size described by the StokesEinstein equation,

$$
D_{0}=\frac{k T}{6 \pi \eta R}
$$

where $k T$ the thermal energy, $\eta$ is the viscosity of solvent, and $R$ is the particle effective radius. Equation (1) and (2) infer that stratified drying may be achieved for evaporation-dominated coating process (high $P_{e}$ ). The particles consolidation front forms at the air-solution interface and grows maximum packing fraction near the surface top. On the other hand, when diffusion dominates (low $P_{e}$ ) particles concentration are predicted to remain dispersed uniformly in the film throughout the coating thickness as it dries (10).

As described in Figure 1, smaller particles with $P_{e}<1$ undergo faster Brownian motion as "kicked" by the solvent molecules, and thus they are able to escape from the descending surface into the bulk film solution during evaporation. On the other hand, the larger particles with $P_{e}>1$ experience much slower Brownian motion unable to escape away from the descending surface. Thus, comparatively heavy particles tend to accumulate and form skin layer at the film surface.

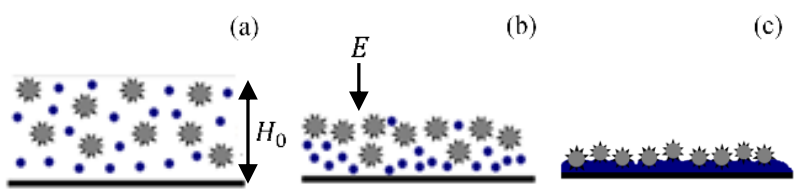

functionalized silica nanoparticles $\bullet$ polymer binder nanoparticles

Figure.1: Schematic diagram shows drying steps of particulate coating, beginning with uniform distribution of particles at the initial time (a). Then evaporation starts and brings particles toward, where larger silica particles are trapped at the surface, while the smaller polymer binder molecules can diffuse away (b). This results in high concentration of silica particles at the film surface, and the polymer binder submerges down to bottom (c)

In this study, low surface energy silica particles with $P_{e}$ (silica) $>1$ was obtained by particle functionalization with fluoroalkylsilane compound, and they was blended with smaller fluorinated polymer binder nanoparticles with $P_{e}$ (polymer) $<1$.
The coating solution was applied on a glass slide substrate by simple drop-casting technique and allowed to dry at different evaporation temperatures. During film drying, self-stratification of the silica particles and fluorinated polymer binder are investigated. The effect of evaporation temperature on stratified particulate coating and the resulting liquid-repellent properties were examined.

\section{Experimental}

\subsection{Formulation of latex-repellent coating}

Fumed silica nanoparticles with primary particle size in a range of 5-25 nm were chemically modified to alter their surface from hydrophilic silanol groups $(-\mathrm{SiOH})$ to hydrophobic fluoroalkyl groups $\left(-\mathrm{CF}_{3}\right)$ by stirring them with $0.2 \mathrm{ml}$ of perfluorooctyltriethoxysilane molecules in a mixture of xylenes for 70-75 $\mathrm{h}$ at room temperature. The silica content in the mixed solvent was $5 \% \mathrm{w} / \mathrm{v}$. Then, polymer binder poly(vinylidene fluoride-co-hexafluoro propylene) (PVDF-HFP) was dissolved in acetone for $0.5 \mathrm{~h}$ at room temperature and added into the silica solution for $2.5 \% \mathrm{w} / \mathrm{v}$ amount. The coating solution was stirred further for $0.5 \mathrm{~h}$ to attain homogeneous formulation.

\subsection{Measurement of solvent evaporation rates}

The evaporation rate $E$ of the solvent was measured by mean of film surface descending rate in unit of $\mathrm{mm} / \mathrm{s}$ by using an ultrasonic sensor for thin film measurement system. The evolution of drying film thickness can be tracked in real time inside a temperature-controlled chamber, in order to study the effect of drying temperature as shown in Figure 2.

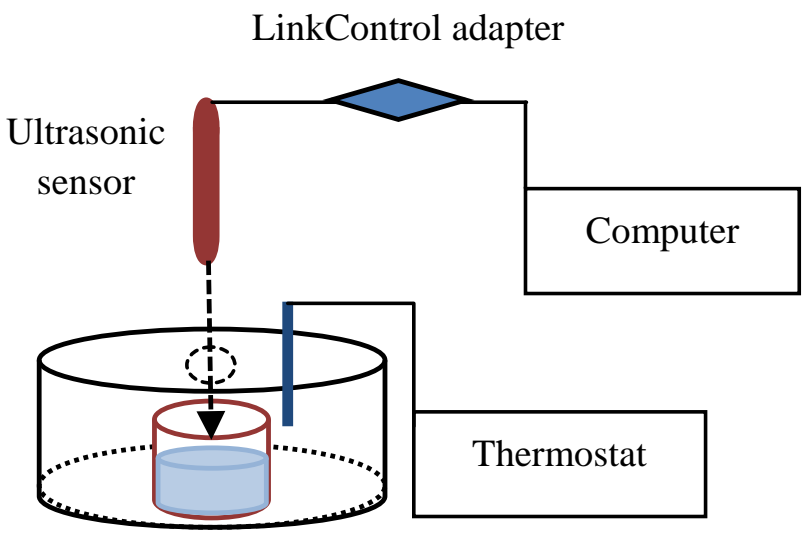

Figure 2: Measurement of film evaporation rates using ultrasonic sensor

\subsection{Contact angles and sliding measurements}

An optical contact angle (CA) measurement system (Dataphysics OCA-15EC) was employed to measure static contact angles and droplet sliding angles (SA). CA measurement was performed using probe liquids of water, and 35\% natural latex with droplet size $\sim 2 \mu \mathrm{L}$ placed at multiple locations on a film surface.

\subsection{Morphology characterizations}

The surface roughness of the film was studied using an atomic force microscope (AFM) and scanning electron microscope 
(SEM), focusing on the effect of the blended polymer binder on the multiscale roughness.

\section{Result and discussion}

The effect of evaporation temperature on the microstructure development of a drying particulate coating was investigated from 1D model of diffusion and convection transport during solvent evaporation. The evaporation rate $E$ was determined from the vertical decrement of film height per unit time, and the values of $E$ from different drying temperatures are shown in Figure 3a. For all cases of drying temperatures, it can be found that the evaporation rate was fast at the initial drying time as the acetone (with low boiling point) predominantly evaporates out of the film. Upon this fast drying, the film surface moved rapidly toward the substrate, causing high rate of convection transport dominated at the beginning, which resulted in the trapping of large silica particles at the surface.
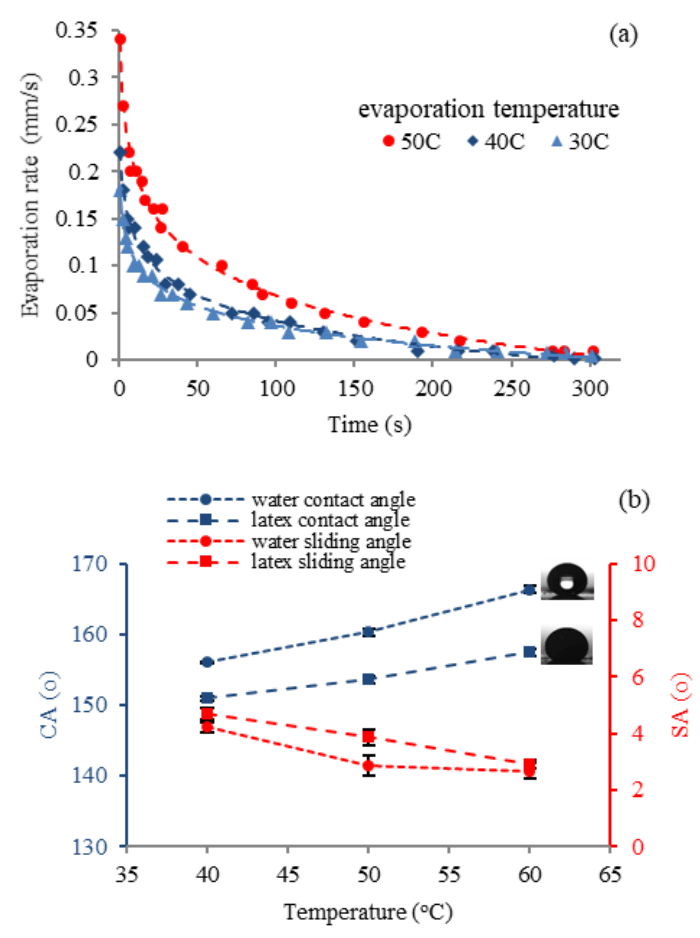

Figure 3: The evaporation rate (a) and the resulting CA and SA values (b) of film dried at temperatures

After $\sim 50 \mathrm{~s}$ of film drying process, the evaporation rates slowed down as the acetone depleted. At this stage, the evaporation rate was controlled by xylene (with high boiling point). This particularly slow evaporation rate near the end of film drying process is actually needed for good coalescence of the polymer binder to prevent film cracking.

It can also be found that higher drying temperature, in general, is associated with more rapid evaporation rate. Once the solvent completely evaporated, highly dense silica particle distribution on the surface can be achieved with the polymer binder located near the substrate interface. The results in Figure $3 \mathrm{~b}$ show that better liquid-repellent properties were obtained for the coated film with higher drying temperature (faster evaporation rate), as larger CA and smaller SA are observed for both water and natural latex droplets.

The detail of the CA and SA of the samples coated with different drying temperature is presented in Table 1 . The superhydrophobic and latex-repellent properties of the coatings improve with faster evaporation rate. The maximum $\mathrm{CA}$ is $166.4^{\circ} \pm 0.6^{\circ}$ and $157.5^{\circ} \pm 0.5^{\circ}$, as well as the minimum sliding angles $2.7^{\circ} \pm 0.3^{\circ}$ and $2.9^{\circ} \pm 0.2^{\circ}$ obtained for water and natural latex respectively.

Table 1: The CA and RA of water and latex

\begin{tabular}{ccccc}
\hline \multirow{2}{*}{$\begin{array}{c}\text { Drying } \\
\text { temp }\end{array}$} & \multicolumn{2}{c}{ Water } & \multicolumn{2}{c}{ Natural latex } \\
\cline { 2 - 5 }$\left({ }^{\circ} \mathrm{C}\right)$ & $\mathrm{CA}\left({ }^{\circ}\right)$ & $\mathrm{SA}\left(^{\circ}\right)$ & $\mathrm{CA}\left(^{\circ}\right)$ & $\mathrm{SA}\left({ }^{\circ}\right)$ \\
\hline 30 & $156.1 \pm 0.1$ & $4.2 \pm 0.2$ & $150.9 \pm 0.3$ & $4.7 \pm 0.2$ \\
40 & $160.3 \pm 0.4$ & $2.9 \pm 0.4$ & $153.7 \pm 0.3$ & $3.9 \pm 0.3$ \\
50 & $166.4 \pm 0.6$ & $2.7 \pm 0.3$ & $157.5 \pm 0.5$ & $2.9 \pm 0.2$ \\
\hline
\end{tabular}

AFM images for surface topography of the coated film were obtained in non-contact mode and the surface profiles of $40^{\circ} \mathrm{C}$ coating condition are presented in Figure. 4. It can be observed for the substantial surface roughness, randomly distributed throughout the studied area, which is a desired properties required for Cassie-Baxter anti-wetting state. The quantitative analysis of surface RMS roughness was determined as $581 \pm 18 \mathrm{~nm}$, which is much larger than the size of the primary silica particles. This result infers that the surface roughness was essentially composed of a larger particle aggregates that diffused slowly and trapped at the surface during the early stage of the film drying.

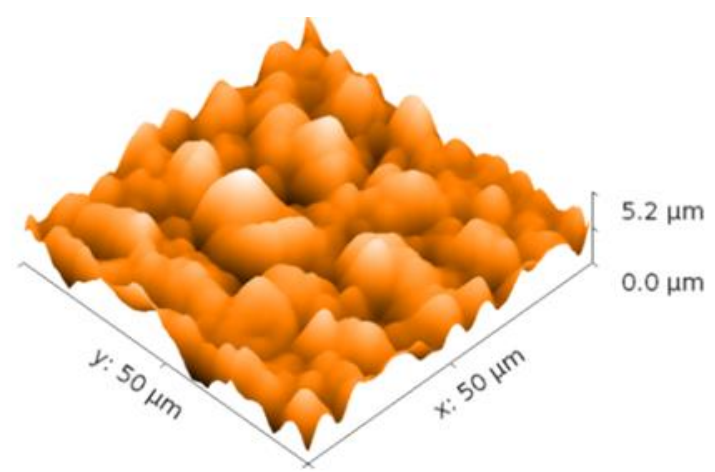

Figure 4: AFM images of film surface structure with $40^{\circ} \mathrm{C}$ drying rate

The film surface morphology of $40{ }^{\circ} \mathrm{C}$ coating condition was further studied using SEM imaging technique, and it clearly revealed hierarchically structured roughness as shown in Figure 5. The overall variation of surface heights was observed at microscale level, featured with numerous nanograins produced by the aggregate of primary silica nanoparticles. This multiscale roughness also rendered small hole size of less than $1 \mu \mathrm{m}$ that can trap countless air pockets on the surface, resulting in a reduction of direct contact between liquid and film surface. This minimizes liquid-film adhesion that corresponds to very low SA values of less than $5^{\circ}$ for both water and natural latex. The SEM crosssection also reveals highly porous structure at the surface produced by silica aggregates, whereas the denser film structure 
can be found below which contains higher ratio of polymer binder that improve the adhesion of the film with substrate. The coated surface exhibits decent liquid-repellent properties even for highly adhesive liquid like natural latex. Figure 6 shows the dynamic wetting properties of water and natural latex droplets sliding on coated surfaces. As revealed by the selected sequential movie frames, it can be observed that the fluid moves easily out of the coated surface without any residual liquid left behind. Moreover, recent studies confirmed that the air pockets underneath the droplets can cause liquid slippery; thus, the liquid flows virtually over a layer of air-solid composite, which significantly reduces surface friction (11). This could find great potential applications in collection and transportation of natural latex liquid with minimum waste and cleaning cost due to adhesion.

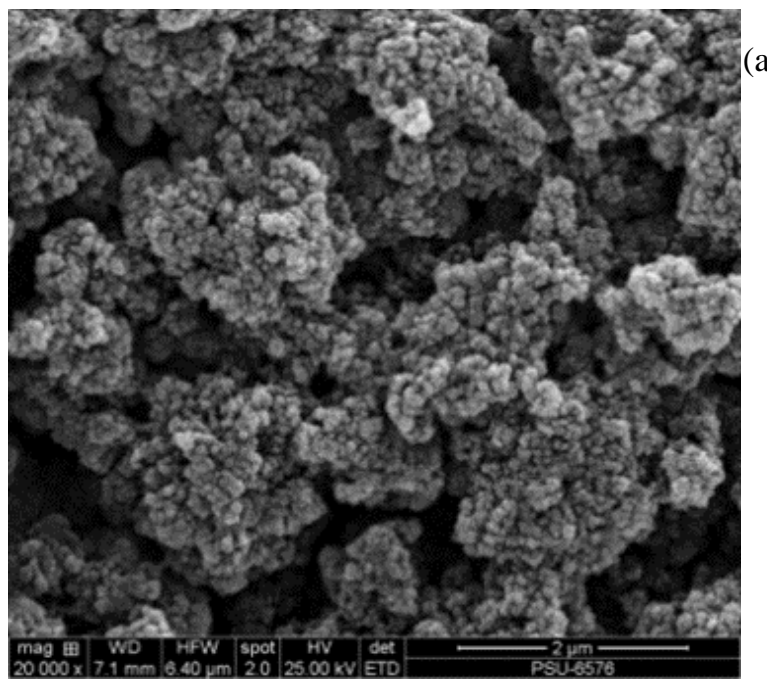

(b)

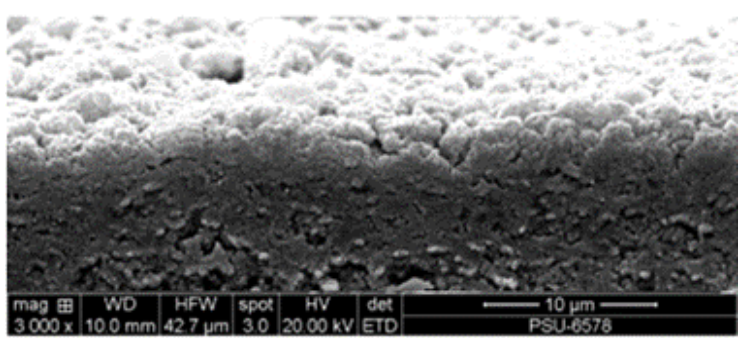

Figure 5: SEM images of stratifying film dried at $40^{\circ} \mathrm{C}$ at top view (a) and cross section (b)

\section{Conclusion}

In summary, we have successfully fabricated extreme antiwetting surface using a solution of surface functionalized silica aggregates blended with PVDF-HFP polymer binder to improve film mechanical durability. During film drying, self-stratification was employed to facilitate vertical phase separation between the silica particles and the polymer binder. The wetting-resistant performance of the resulting films, dried with different evaporation rates controlled by varied temperatures, was studied. It was found that liquid-repellent properties significantly improved with increasing coating temperature i.e. at high evaporation rate. This suggests that silica particles were trapped more at the surface when the convection transport dominated. It can be clearly seen high degree of surface roughness from AFM and SEM even for the film that was incorporated with significant amount of polymer binder. Moreover, the coated surface also shows good superhydrophobic and latex-repellent properties with high CA and low SA. It is expected that this simple single-step coating technique may possibly open a new way to improve the mechanical durability of superhydrophobic and latex-repellent surfaces for practical applications.
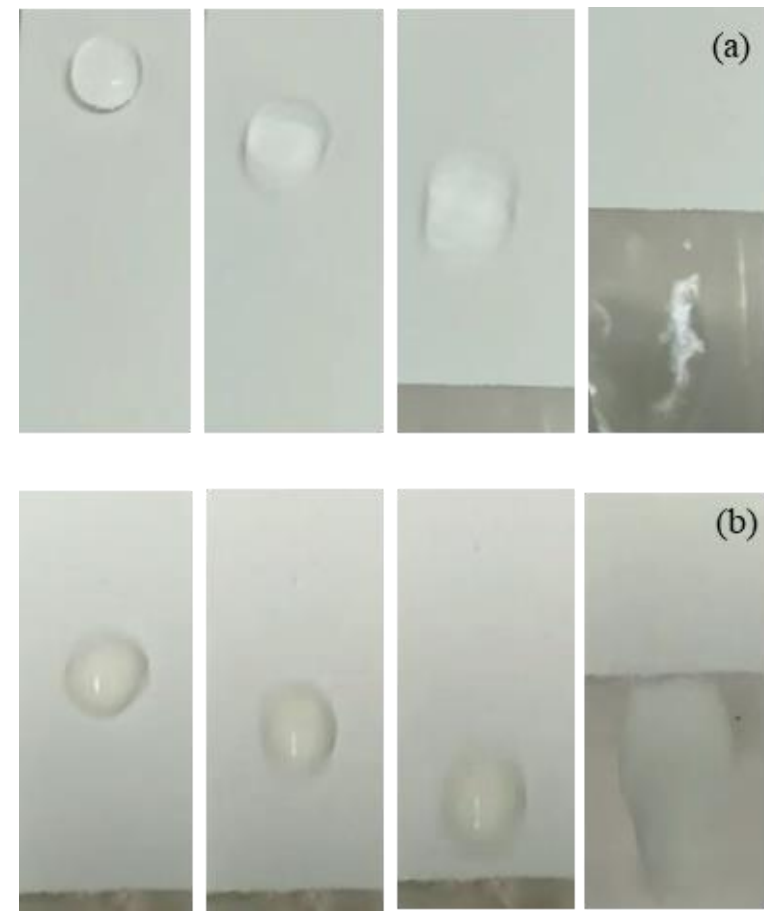

Figure 6: Selected movie frames display the flow of water (a), and latex droplets (b) out of coated surface without leaving any residue

\section{Aknowledgment}

The authors gratefully acknowledge the Center of Excellence in Nanotechnology for Energy (CENE), the Department of Physic, Faculty Science, Prince of Songkla University and the Department of Research and Development, Faculty of Science and Technology, Fatoni University for providing the facilities of laboratory. The financial support from PSU-Ph.D. Scholarship and Research Grant for Thesis, by Graduate School, Prince of Songkla University.

\section{Ethical issue}

Authors are aware of, and comply with, best practice in publication ethics specifically with regard to authorship (avoidance of guest authorship), dual submission, manipulation of figures, competing interests and compliance with policies on research ethics. Authors adhere to publication requirements that submitted work is original and has not been published elsewhere in any language.

\section{Competing interests}

The authors declare that there is no conflict of interest that would prejudice the impartiality of this scientific work. 


\section{Authors' contribution}

S. Hajeesaeh and S. Kariy were responsible for data collection, data analyses and manuscript writing. C. Daengnam and N. Muensit initiated experimental design, performed data analysis and edited manuscript.

\section{References}

1. Arcos D, Fal-Miyar V, Ruiz-Hernández E, Garcia-Hernández M, RuizGonzález ML, González-Calbet J, et al. Supramolecular mechanisms in the synthesis of mesoporous magnetic nanospheres for hyperthermia. Journal of Materials Chemistry. 2012;22(1):64-72.

2. Nosonovsky M. Multiscale Roughness and Stability of Superhydrophobic Biomimetic Interfaces. Langmuir. 2007;23(6):3157-61.

3. Chunglok A, Muensit N, Daengngam C. Extreme Wetting-Resistant Multiscale Nano-/Microstructured Surfaces for Viscoelastic Liquid Repellence. J Nanomater. 2016;2016:13.

4. Routh AF. Drying of thin colloidal films. Reports on Progress in Physics. 2013;76(4):046603.

5. Trueman RE, Lago Domingues E, Emmett SN, Murray MW, Routh AF. Auto-stratification in drying colloidal dispersions: A diffusive model. 2012;377(1):207-12.

6. Routh AF, Russel WB. Deformation Mechanisms during Latex Film Formation: Experimental Evidence. Industrial \& Engineering Chemistry Research. 2001;40(20):4302-8.

7. Ensikat HJ, Ditsche-Kuru P, Neinhuis C, Barthlott W. Superhydrophobicity in perfection: the outstanding properties of the lotus leaf. Beilstein journal of nanotechnology. 2011;2:152-61.

8. Routh AF, Zimmerman WB. Distribution of particles during solvent evaporation from films. 2004;59(14):2961-8.

9. Cardinal CM, Jung YD, Ahn KH, Francis LF. Drying regime maps for particulate coatings. AIChE Journal. 2010;56(11):2769-80.

10. Vanderhoff JW, Bradford EB, Carrington WK. The transport of water through latex films. Journal of Polymer Science: Polymer Symposia. 1973;41(1):155-74.

11. Kota AK, Li Y, Mabry JM, Tuteja A. Hierarchically Structured Superoleophobic Surfaces with Ultralow Contact Angle Hysteresis. Advanced Materials. 2012;24(43):5838-43. 